\title{
PREFERÊNCIAS DE ATIVIDADE FÍSICA E ESPORTES PARA ESCOLARES NO ENSINO FUNDAMENTAL
}

DOI: $105902 / 0102830822291$

Data de submissão: 24-05-2016

Data de Aceite: 01-07-2016

Vanilson Batista Lemes

Universidade Federal do Rio Grande do Sul vanilson.lemes@hotmail.com

Caroline Brand Universidade Federal do Rio Grande do Sul carolbrand@hotmail.com.br

Arieli Fernandes Dias Universidade Federal do Rio Grande do Sul ariieli_dias@hotmail.com

Rodrigo Baptista Moreira Universidade Luterana do Brasil - São Jerônimo rbmoreira2@gmail.com

Adroaldo Gaya
Universidade Federal do Rio Grande do Sul
adroaldogaya@hotmail.com

Anelise Gaya

Universidade Federal do Rio Grande do Sul anegaya@gmail.com

Resumo: Objetivo: Descrever as preferências por atividade física e esportes de escolares do sexto ao oitavo ano. Metodologia: Estudo descritivo com abordagem quantitativa realizado com 52 escolares, sendo 28 meninos, questionados através de um instrumento semiestruturado. Resultados: As modalidades que os estudantes têm preferência em praticar foram lutas $(53,8 \%)$, voleibol $(38,5 \%)$ e futsal $(30,8 \%)$. Por gênero, as preferências foram lutas $(100 \%)$ e futsal $(46,4 \%)$ para os meninos e voleibol $(62,5 \%)$ e dança $(45,8 \%)$ para as meninas. Conclusão: Há divergência entre as práticas de AF e esportes realizadas e aquilo que os escolares preferem.

Palavras-Chave: Planejamento. Educação Física. Saúde 


\section{Introdução}

A necessidade de realização do presente estudo se originou de um discurso repetido em algumas realidades escolares: "as crianças e os jovens de hoje só gostam de futebol, ou tão pouco gostam disso, as meninas principalmente já não gostam de mais nada, só querem saber de ficar sentadas mexendo no celular durante as aulas de Educação Física (EF). Essa fala foi relatada ao longo de conversas informais com docentes de EF no interior do Rio Grande do Sul. Cada vez que esse fato se repetia, nos deparávamos com algumas dúvidas: (1) havia ensino de atividades além do futebol nessas aulas de EF? (2) os professores estariam cumprindo seu papel em ensinar diferentes formas de atividade física $(\mathrm{AF})$, modalidades esportivas e expressões da cultura corporal do movimento? (3) as aulas de EF oferecidas a essas crianças eram condizentes com as preferências dos alunos e as suas necessidades?

Parte dessas questões é abordada em alguns estudos nacionais e internacionais, que demonstraram pouca eficiência da EF no que corresponde a qualidade de conteúdo, baixa quantidade de AF organizada, falta de comprometimento docente e pouca variedade de esportes ensinados que não iam além do futebol desorganizado (DE OLIVEIRA FORTES et al., 2012; HINO et al., 2010; KAHAN; MCKENZIE, 2015; KREMER; REICHERT; HALLAL, 2012). Para superar tal conjuntura, entendemos que a EF deve oportunizar aulas organizadas e variadas, considerando o desenvolvimento das habilidades motoras, o ensino do esporte, objetivando o ensino aprendizagem para a saúde, lazer e desempenho (GAYA et al., 2008; GAYA, 2009). A EF também deve proporcionar a inclusão de todos os alunos em momentos ativos, para que desfrutem dos benefícios que a prática regular de AF e esportes pode gerar, como o atingimento de níveis de AF adequados e a manutenção da aptidão física, protegendo os escolares contra doenças, aumentando o bem-estar, a integração entre os indivíduos, propiciando também chances para uma vida ativa e saudável na idade adulta (BARNEKOW-BERGKVIST et al., 2001; DENTON et al., 2013; EKELUND et al., 2006; GAYA et al., 2010, 2008; GAYA, 2009; MOSER et al., 2013; SAVOLAINEN et al., 2015; SHAPE, 2013; UNESCO, 2015).

Considerando a perspectiva de EF escolar apresentada, a fala dos professores sobre 
o que os seus alunos preferem é no mínimo passível de questionamento pois demonstra um certo descaso com a prática docente. Ao invés de apenas observar e discursar sobre, pensamos que essas preferências devem ser investigadas. Isso se justifica para que se possa além de ensinar expressões da cultura corporal pré-determinadas pelos currículos em EF, se consiga também elaborar aulas que vão ao encontro dos interesses dos alunos, aumentando a adesão destes e consequentemente realizar aulas de boa qualidade para todos. Dessa forma, o objetivo geral do presente estudo foi descrever as preferências por AF e esportes de escolares do sexto ao oitavo ano, sob quatro campos de análise: (1) os gostos e os motivos; (2) o que já foi realizado; (3) as práticas de preferência; e (4) as preferências de acordo com o gênero.

\section{Metodologia}

Estudo descritivo com abordagem quantitativa. Os escolares foram selecionados por conveniência, em uma Escola Estadual de Ensino Fundamental no Município de Charqueadas, Rio Grande do Sul, devido a instituição de ensino ser o local onde um dos investigadores lecionava. Desse modo, participaram do estudo voluntariamente 52 alunos, destes 28 meninos e 24 meninas, dos 11 aos 14 anos de idade das turmas de $6^{\circ}, 7^{\circ}$ e $8^{\circ}$ ano do ensino fundamental. As informações referentes a identidade foram mantidas sob sigilo e o termo de consentimento livre e esclarecido foi assinado pelos pais ou responsáveis. 0 presente estudo foi aprovado pelo Comitê de Ética em Pesquisa com Seres Humanos da Universidade Federal do Rio Grande do Sul sob parecer número 259.883.

O instrumento utilizado para a pesquisa foi um questionário semiestruturado com perguntas fechadas e abertas, elaborado de acordo com observações de campo realizadas pelo investigador, as perguntas foram divididas em: (1) você gosta de praticar atividades físicas e esportes? (sim ou não); se você não gosta, resuma aqui o motivo (resposta aberta); (2) qual das modalidades de AF e esporte você já praticou dentro ou fora das aulas de EF? Marque um $\mathrm{X}$ em todas que você já fez: futsal, handebol, voleibol, basquetebol, atletismo, caminhada orientada, dança, ginastica, lutas, jogos recreativos, circuitos motores; e (3) cite três modalidades de AF e esporte que você prefere praticar (resposta aberta). 
Para o tratamento dos dados foram utilizadas análises descritivas, ilustradas em gráficos de barra com os valores percentuais e de frequência. As perguntas 1 e 3 de caráter aberto foram analisadas da seguinte forma: (1) descrevemos de forma textual os motivos reportados pelos estudantes que os levavam a não praticar AF e esportes; (2) categorizamos as respostas referentes ao que os estudantes teriam preferência em praticar, apresentadas por modalidades com valores absolutos e percentuais e também divididos por gênero. Todos os dados foram tratados nos programas SPSS for Windows versão 20.0 e no graphpad prism versão 5.0 .

\section{Resultados}

As respostas sobre gostar de praticar de AF e esporte, assim como os motivos que levam os estudantes a não praticar são demonstrados no quadro 1.

Quadro 1 - Ocorrência do gosto e os motivos para a prática de atividades físicas e esporte

\begin{tabular}{|c|c|c|}
\hline $\begin{array}{c}\text { Você gosta de praticar } \\
\text { atividade física e esporte? }\end{array}$ & N (\%) & $\begin{array}{c}\text { Motivos porque você não gosta de praticar atividade } \\
\text { física e esporte? }\end{array}$ \\
\hline Sim & $44(84,6)$ & - \\
\hline Não & $8(15,4)$ & $\begin{array}{c}\text { Preguiça, não gostar de jogar, não saber jogar, não gostar } \\
\text { de excícios e não gostar de praticar esportes em função } \\
\text { da pouca habilidade quando comparado aos colegas. }\end{array}$ \\
\hline
\end{tabular}

\%: valor proporcional; $\mathrm{N}$ : valor absoluto; * 2 meninos e 6 meninas 
A figura 1 apresenta a descrição da ocorrência de AF e esportes já praticados pelos estudantes.

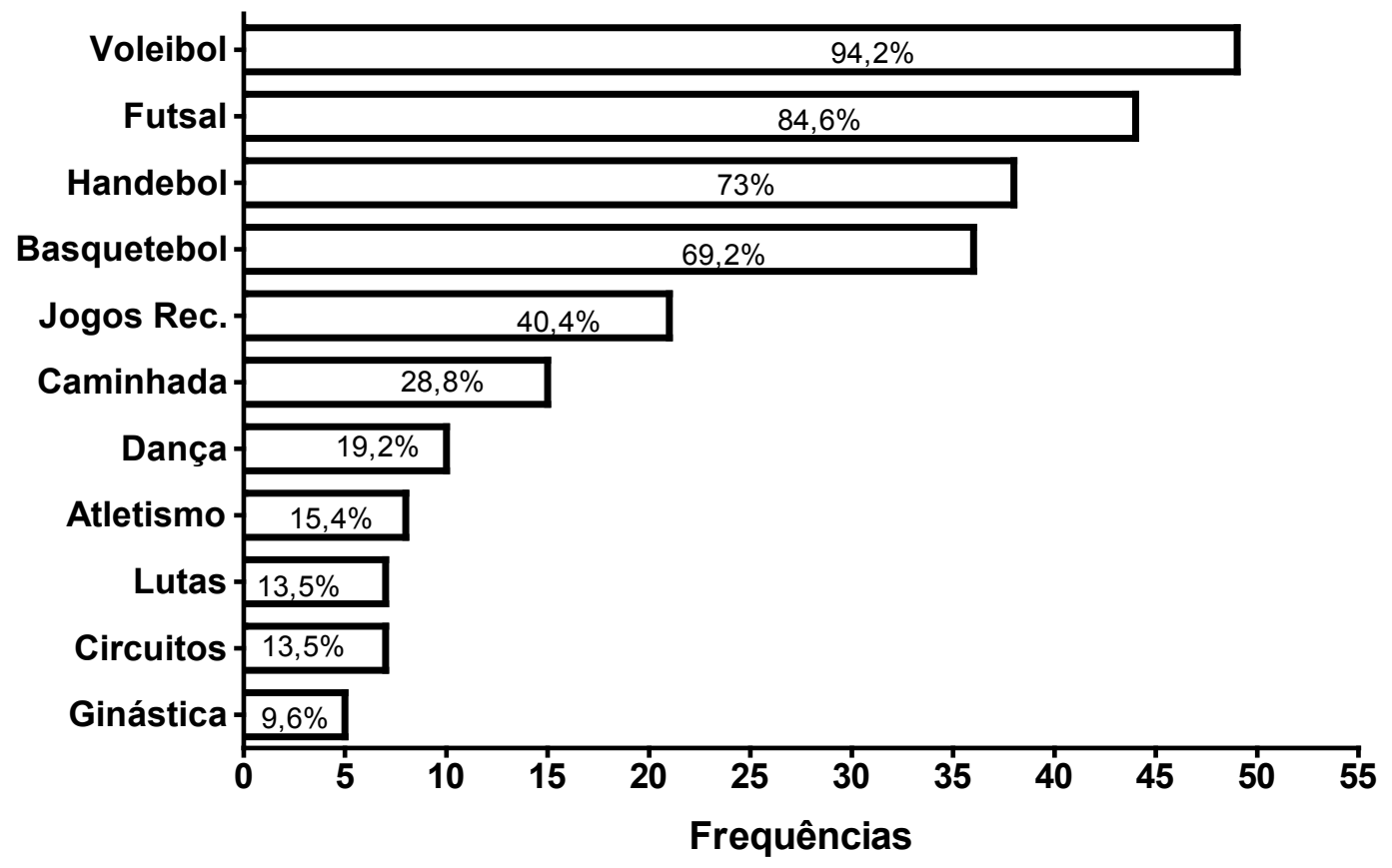

Figura 1. Ocorrência de atividades físicas e esportes já praticados pelos estudantes.

A figura 2 demonstra a ocorrência de atividades físicas e esportes que os estudantes tem preferência em praticar. Foram encontradas ocorrências em torno de $10 \%$ dos esportes "não nacionais" como preferência dos alunos, como o baseball e o futebol americano, superando o interesse pela prática do futebol.

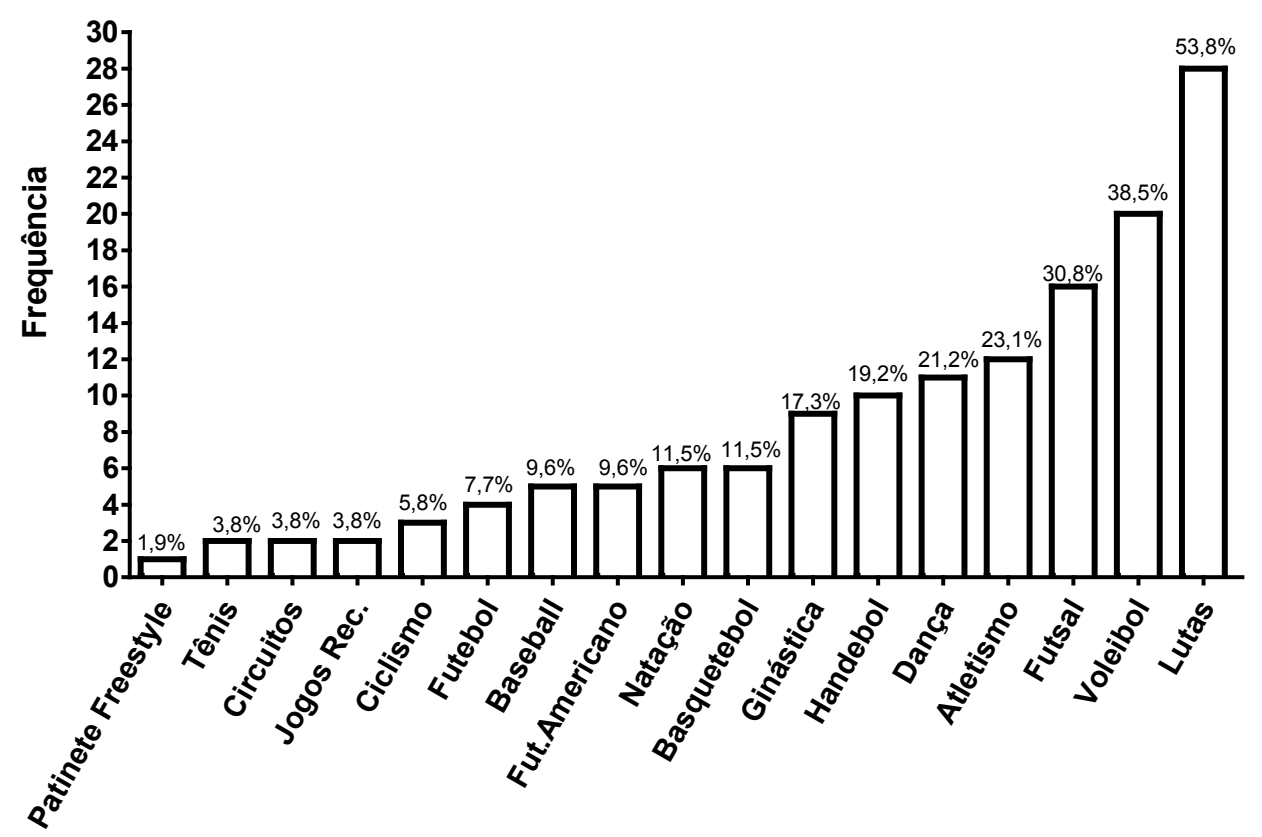

Figura 2. Ocorrência das preferências de atividades físicas e esportes. 
A tabela 1 demonstra a distribuição das preferências das modalidades de acordo com o gênero. Observamos que as lutas e o futsal são a preferência dos meninos e o voleibol e a dança para as meninas. Também podemos perceber que as meninas não demonstraram interesse pela prática de outras modalidades além de voleibol, futsal, dança, handebol e ginástica.

Tabela 1. Distribuição de preferências para aprendizagem por gênero

\begin{tabular}{cccc}
\hline Masculino & Feminino & Total \\
Modalidades & $(\mathrm{n}=28)$ & $(\mathrm{n}=24)$ & $\mathbf{n}=52)$ \\
& $\mathbf{N}(\%)$ & $\mathbf{N}(\mathbf{\%})$ & $28(53,8 \%)$ \\
\hline Lutas & $28(100 \%)$ & - & $20(38,5 \%)$ \\
Voleibol & $5(17,8 \%)$ & $15(62,5 \%)$ & $16(30,8 \%)$ \\
Futsal & $13(46,4 \%)$ & $3(12,5 \%)$ & $12(42,8 \%)$ \\
Atletismo & $12(42,8 \%)$ & - & $11(21,2 \%)$ \\
Dança & - & $11(45,8 \%)$ & $10(19,2 \%)$ \\
Handebol & $3(10,7 \%)$ & $7(29,1 \%)$ & $9(17,3 \%)$ \\
Ginástica & - & $9(32,1 \%)$ & $37(71,1 \%)$ \\
Outros* & $37(75,6 \%)$ & - &
\end{tabular}

n: número de sujeitos; N: quantidade absoluta de respostas; \%: valor percentual; * Outros: Soma das respostas do Basquetebol, natação, futebol americano, baseball, futebol, ciclismo, jogos recreativos, circuitos, tênis, patinete freestyle.

\section{DISCUSSÃO}

Esse estudo teve como objetivo descrever as preferências por AF e esportes de escolares do sexto ao oitavo ano, sob quatro campos de análise: (1) os gostos e os motivos; (2) o que já foi realizado; (3) as práticas de preferência; e (4) as preferências de acordo com o gênero. Os resultados demonstraram que $84,6 \%$ dos escolares gostam de praticar AF e esportes. Dos 8 alunos que responderam não gostar, as justificavas foram a preguiça, falta de domínio sobre a atividade ou por não gostarem de praticar atividades com outros escolares que tem maior domínio.

Embora, a maioria dos escolares tenham relatado gostar de praticar AF e esporte, a literatura apresenta altos índices de crianças e adolescentes que não atingem o nível mínimo de AF recomendado para a saúde o qual poderia ser obtido através dessas 
práticas, além de altas ocorrências de sedentarismo e excesso de peso nesta faixa etária (FLORES et al., 2013; GORDON-LARSEN; NELSON; POPKIN, 2004; HINO et al., 2010; KAHAN; MCKENZIE, 2015; KREMER; REICHERT; HALLAL, 2012). Esses fatos quando confrontados com os nossos resultados nos deixam intrigados pois, se a maior parte dos escolares reportou gostar desses conteúdos, porque há tão pouca AF e esporte realizados? Isso se expande em um problema bastante amplo, não só no Brasil, mas em um nível global (HALLAL et al., 2012).

Tais presunções nos levam a refletir sobre localidades específicas como a região sul do Brasil, onde os dados de um recente estudo mostraram que há neste local uma maior proporção de pessoas que reportaram não gostar de praticar AF ou algum esporte (ESPORTE, 2015). Esse resultado vem ao encontro dos nossos, e pode ser atribuído aos aspectos intrínsecos para a prática de AF ou esporte, como a preguiça, junto das barreiras, como as climáticas, estruturais e de ambiente (WANG et al., 2016). Esses motivos, são apresentados também em outras pesquisas, como em $1 / 3$ da população adolescente de Pelotas-RS que apresentou a falta de tempo, companhia, local adequado, dinheiro, preguiça/cansaço e os dias de chuva como barreiras à AF (COPETTI; NEUTZLING; SILVA, 2010). Os fatores internos como a preguiça se relacionam com a motivação, que parece ser diferente em meninos e meninas, onde o prazer pelo movimento e o gosto pelo esporte favorecem os meninos, já a distração e o divertimento é determinante para a prática de AF e esporte das meninas (BERLEZE; VIEIRA; KREBS, 2008).

Os nossos resultados, aproximam-se de valores encontrados para a população brasileira, no que se refere as relações da prática de esporte, AF e o gênero (ESPORTE, 2015). As atividades esportivas mais praticadas no país, foram o futebol pelo gênero masculino com $66,2 \%$ e o voleibol e futebol para o feminino com $20,5 \%$ e $19,2 \%$ respectivamente (ESPORTE, 2015). Fica evidente também em nossos dados, uma ampla diversidade reportada pela preferência em realizar algum tipo de AF e/ou esporte principalmente para os meninos, sendo que eles citaram 18 diferentes modalidades. Por outro lado, conforme já discutido acima, 8 escolares relataram não gostar de praticar AF e/ou esportes. Esses resultados e diferenças demonstram que a AF e o esporte são comportamentos humanos complexos e dependentes de diversos fatores intervenientes (SALLIS et al., 2006; WANG 
et al., 2016).

Não obstante, as pesquisas levantam hipóteses isoladas que a ineficácia das aulas de EF pode ser atribuída à falta de estrutura, condições materiais e ambientes adequados (SALLIS et al., 2006). O que também é constantemente relatado por professores no diaa-dia escolar como barreira para boas aulas. Além disso, o estilo de vida atual favorece o sedentarismo (BAILEY et al., 2012; MAHER et al., 2014), o que dificulta o envolvimento dos estudantes em outras culturas além das mais difundidas no país, como o futebol. Isso se associa a fala dos docentes e pôr fim ao conteúdo das aulas que não contempla os tipos de AF preferidos dos escolares, as suas individualidades biológicas e os fatores socioculturais (GONÇALVES et al., 2007; LYTLE et al., 2009).

No presente estudo observamos que há uma desarmonia no que se refere à preferência em praticar e o que os alunos efetivamente já tinham realizado em AF e esporte, o que provavelmente é determinado pelas limitadas oportunidades das práticas que são ofertadas para crianças e jovens na EF escolar (GAYA, 2009; SHAPE, 2013). Os estudantes avaliados realizaram mais esportes coletivos e com bola, do que outras modalidades, como a dança, a ginástica e o atletismo. De encontro a essa relação dicotômica, aquilo que mais gostariam de praticar foram modalidades individuais como as lutas e o atletismo para os meninos, embora ainda mantenham a preferência na prática de esportes coletivos tradicionais, como o futsal. Com relação as meninas, o voleibol, a ginástica e a dança apareceram como modalidades que gostariam de praticar. Também foram citadas atividades físicas e esportes bastante distintos e com pouca adesão no Brasil (ESPORTE, 2015), como o futebol americano, o ciclismo, o baseball e a natação.

Tais resultados demonstraram que é importante incluir alternativas aos esportes tradicionais, no sentido de aumentar a motivação dos estudantes para aderirem às aulas de EF escolar (MEARS, 2010). Proporcionando assim, oportunidade de conhecimento variado e aumento dos níveis de AF (BARNEY; PRUSAK, 2015). Nesse sentido, estudos demonstraram que a oferta de modalidades distintas, por exemplo, um jogo de Rugby adaptado, parecem ser eficientes, principalmente quando há engajamento em jogos competitivos e entre times (WOOD; HALL, 2015). Bem como, o freesbie e a caminhada ao som de música parecem gerar maiores níveis de AF em estudo realizado nos Estados Unidos (BARNEY; PRUSAK, 
2015). Logo, estas diversidades ofertadas na EF além de proporcionar benefícios para a saúde, poderá suprir a amplitude de necessidades e preferências das crianças e jovens.

Esses pressupostos servem como sugestões para elaboração de estratégias para o ensino em EF mais eficiente. Todavia, encontramos limitações na presente investigação, como a transversalidade que pode gerar diferenças nas respostas conforme o avanço da maturidade dos escolares. Além disso, há tendência de os escolares superestimarem o número de modalidades respondidas no questionário para impressionarem o professor de educação física. Também há limitação no instrumento de pesquisa com relação aos conceitos estabelecidos a priori pelos escolares sobre o que é esporte ou AF, para tanto consideramos ambos os termos, afim de abranger uma gama maior de atividades e modalidades. Isso gerou um obstáculo na identificação exata, se a prática era o esporte, jogo ou uma AF derivada do esporte, junto da não diferenciação se a modalidade era praticada na aula de EF ou fora dela.

Concluímos que a maioria dos sujeitos avaliados gosta de praticar AF ou esporte, relataram com maior frequência o voleibol e futsal como atividades já realizadas. Fato que diverge em parte com o que eles prefeririam praticar. As lutas e futsal são preferências, não exclusivas, para os meninos e o voleibol, dança e ginástica para as meninas. Sugerimos através do nosso estudo, que as aulas de EF escolar devem ser contextualizadas para as crianças e jovens de acordo com as preferências para os diferentes gêneros e suas capacidades físicas. Bem como, indicamos que outros estudos façam a relação estatística entre as preferências dos escolares, os níveis de AF, a aptidão física, marcadores fisiológicos de saúde e motivação dos professores para planejar as aulas, afim de atender crianças e jovens no que se refere às suas necessidades psicossociais e de promoção da saúde através da EF escolar. 


\section{Referências}

BAILEY, D. P. et al. Accelerometry-assessed sedentary behaviour and physical activity levels during the segmented school day in 10-14-year-old children: The HAPPY study. European Journal of Pediatrics, v. 171, n. 12, p. 1805-1813, 2012.

BARNEKOW-BERGKVIST, M. et al. Adolescent determinants of cardiovascular risk factors in adult men and women. Scandinavian Journal of Public Health, v. 29, n. 3, p. 208-217, Sep. 2001.

BARNEY, D.; PRUSAK, K. A. Effects of Music on Physical Activity Rates of Elementary Physical Education Students. The physical educator, v. 72, p. 236-244, 2015.

BERLEZE, A.; VIEIRA, L.; KREBS, R. Motivos que levam crianças à prática de atividades motoras na escola. Revista da Educação Física/UEM, v. 13, n. 1, p. 99-107, 2008.

COPETTI, J.; NEUTZLING, M. B.; SILVA, M. C. Barreiras à prática de atividades físicas em adolescentes de uma cidade do sul do Brasil. Revista Brasileira de Atividade Física e Saúde, v. 15, n. 2, p. 88-94, 2010.

DE OLIVEIRA FORTES, M. et al. A educação física escolar na cidade de pelotas-rs: Contexto das aulas e conteúdos. Revista da Educacao Fisica, v. 23, n. 1, p. 69-78, 2012.

DENTON, S. J. et al. Cardiorespiratory Fitness Is Associated with Hard and Light Intensity Physical Activity but Not Time Spent Sedentary in 10-14 Year Old Schoolchildren: The HAPPY Study. PLoS ONE, v. 8, n. 4, 2013.

EKELUND, U. et al. TV viewing and physical activity are independently associated with metabolic risk in children: The European youth heart study. PLoS Medicine, v. 3, n. 12, p. 2449-2457, 2006. 
ESPORTE, Ministério do. Diagnóstico Nacional do Esporte: caderno 1, 2015.

FLORES, L. S. et al. Trends of underweight, overweight, and obesity in Brazilian children and adolescents. Jornal de Pediatria, v. 89, n. 5, p. 456-461, 2013.

GAYA, A. R. et al. Association of leisure time physical activity and sports competition activities with high blood pressure levels: Study carried out in a sample of portuguese children and adolescents. Child: Care, Health and Development, v. 37, n. 3, p. 329-334, 2010.

GAYA, A. Sobre o esporte para crianças e jovens. Movimento (ESEF/UFRGS), v. 6, n. 13, p. 1-14, 2009.

GAYA, A. C. A. et al. Ciências do movimento humano. Introdução à metodologia da pesquisa. 1. ed. Porto Alegre: Artmed, 2008.

GONÇALVES, H. et al. Fatores socioculturais e nível de atividade física no início da adolescência. Revista Panamericana de Salud Pública, v. 22, n. 4, p. 246-253, 2007.

GORDON-LARSEN, P.; NELSON, M. C.; POPKIN, B. M. Longitudinal physical activity and sedentary behavior trends: Adolescence to adulthood. American Journal of Preventive Medicine, v. 27, n. 4, p. $277-283,2004$.

HALLAL, P. C. et al. Global physical activity levels: Surveillance progress, pitfalls, and prospects. The Lancet, v. 380, n. 9838, p. 247-257, 2012.

HINO, A. A. F. et al. Observação Dos Níveis De Atividade Física, Contexto Das Aulas E Comportamento Do Professor Em Aulas De Educação Física Do Ensino Médio Da Rede Pública. Revista Brasileira De Atividade Física \& Saúde, v. 1, n. 1, p. 21-30, 2010. 
KAHAN, D.; MCKENZIE, T. L. The Potential and Reality of Physical Education in Controlling Overweight and Obesity. American Journal of Public Health, v. 105, n. 4, p. 4-11, 2015.

KREMER, M. M.; REICHERT, F. F.; HALLAL, P. C. Intensidade e duração dos esforços físicos em aulas de Educação Física. Revista de Saúde Pública, v. 46, n. 2, p. 320-326, 2012.

LYTLE, L. A. et al. Mediators affecting girls' levels of physical activity outside of school: Findings from the trial of activity in adolescent girls. Annals of Behavioral Medicine, v. 38, n. 2, p. 124-136, 2009.

MAHER, C. et al. Reconsidering the sedentary behaviour paradigm. PLoS ONE, v. 9, n. 1, 2014.

MEARS, D. Physical Education Waivers and Young Adult Activity. Physical Educator, v. 67, n. 2, p. 90-101, 2010.

MOSER, D. C. et al. Anthropometric measures and blood pressure in school children. Jornal de Pediatria, v. 89 , n. 3, p. 243-249, 2013.

SALLIS, J. F. et al. an Ecological Approach To Creating Active Living Communities. Annual Review of Public Health, v. 27, n. 1, p. 297-322, 2006.

SAVOLAINEN, J. et al. Decreasing cholesterol levels in the community - lifestyle change with statin? BMC Family Practice, v. 16, n. 1, p. 1-8, Mar. 2015.

SHAPE. Position Statement. Comprehensive School Physical Activity Programs: Helping All Students Achieve 60 Minutes of Physical Activity Each Day.Society of Health and Physical Educators, 2013. Disponível em: <http://www.shapeamerica.org/advocacy/positionstatements/pa/upload/CSPAP-PositionStatement-2.pdf>

UNESCO. Diretrizes em educação física de qualidade (EFQ) para Gestores de Políticas. Brasília, 2015. Disponível em: <http://unesdoc.unesco.org/images/0023/002319/231963POR.pdf> 
WANG, Y. et al. A review on the effects of physical built environment attributes on enhancing walking and cycling activity levels within residential neighborhoods. Cities, v. 50, p. 1-15, 2016.

WOOD, C.; HALL, K. Physical education or playtime: which is more effective at promoting physical activity in primary school children? BMC Research Notes, v. 8, n. 1, p. 8-12, 2015. 


\section{PHYSICAL ACTIVITY AND SPORTS PREFERENCES TO STUDENTS ON ELEMENTARY EDUCATION}

\section{Abstract}

Aim: To describe preferences for physical activity and school sports the sixth to eighth year. Methodology: descriptive study with a quantitative approach carried out with 52 students, 28 boys, asked through a semi-structured instrument. Results: The procedure by which students are preferred in practice were combats (53.8\%), volleyball (38.5\%) and futsal $(30.8 \%)$. By gender, preferences were combats $(100 \%)$ and futsal $(46.4 \%)$ for boys and volleyball $(62.5 \%)$ and dance $(45.8 \%)$ for girls. Conclusion: There is difference between AF and held sports practices and what the students prefer.

Keywords: Planning. Physical Education. Health

\section{PREFERENCIAS DE LAACTIVIDAD FÍSICA Y DEPORTES PARAALUMNOS EN LA ESCUELA ELEMENTAL}

Resumen

Objetivo: Describir las preferencias para los deportes y actividad física (AF) en la escuela del sexto al octavo año. Metodología: estudio descriptivo con un enfoque cuantitativo llevado a cabo con 52 estudiantes, 28 niños, interrogados por uno instrumento semiestructurado. Resultados: Los estudiantes prefieren em la práctica las luchas $(53,8 \%)$, voleibol $(38,5 \%)$ y fútbol sala $(30,8 \%)$. Por género, las preferencias fueron luchas $(100 \%)$ y fútbol sala $(46,4 \%)$ de los niños y de voleibol $(62,5 \%)$ y la danza $(45,8 \%)$ para las niñas. Conclusión: Hay diferencia entre la AF y las prácticas deportivas llevados a cabo y lo que los estudiantes prefieren.

Palabras-clave: Planificación. Educación Física.Salud 